\title{
The association between cardiac physiology, acquired brain injury, and postnatal brain growth in critical congenital heart disease
}

\author{
Shabnam Peyvandi, MD, ${ }^{\text {a }}$ Hosung Kim, PhD, ${ }^{\mathrm{d}}$ Joanne Lau, MS, ${ }^{\mathrm{b}}$ A. James Barkovich, MD, \\ Andrew Campbell, MD, ${ }^{\mathrm{e}}$ Steven Miller, MD, MAS, ${ }^{\mathrm{f}}$ Duan $\mathrm{Xu}, \mathrm{PhD},{ }^{\mathrm{b}}$ and Patrick McQuillen, $\mathrm{MD}^{\mathrm{c}}$
}

\section{ABSTRACT}

Objective: To assess the trajectory of perioperative brain growth in relationship to cardiac diagnosis and acquired brain injuries.

Methods: This was a cohort study of term neonates with hypoplastic left heart syndrome (HLHS) and d-transposition of the great arteries (d-TGA). Subjects underwent magnetic resonance imaging of the brain pre- and postoperatively to determine the severity of brain injury and total and regional brain volumes by the use of automated morphometry. Comparisons were made by cardiac lesion and injury status.

Results: A total of 79 subjects were included (49, d-TGA; 30, HLHS). Subjects with HLHS had more postoperative brain injury (55.6\% vs 30.4\%,P $=.03)$ and more severe brain injury (moderate-to-severe white matter [WM] injury, $P=.01$ ). Total and regional perioperative brain growth was not different by brain injury status (either pre- or postoperative). However, subjects with moderate-to-severe WM injury had a slower rate of brain growth in WM and gray matter compared with those with no injury. Subjects with HLHS had a slower rate of growth globally and in WM and deep gray matter as compared with d-TGA (total brain volume: $12 \mathrm{~cm}^{3} /$ wk vs $7 \mathrm{~cm}^{3}$; WM: $2.1 \mathrm{~cm}^{3} /$ wk vs $0.6 \mathrm{~cm}^{3}$; deep gray matter: $1.5 \mathrm{~cm}^{3} /$ wk vs $0.7 \mathrm{~cm}^{3} ; P<.001$ ), after we adjusted for gestational age at scan and the presence of brain injury. This difference remained after excluding subjects with moderate-to-severe WM injury.

Conclusions: Neonates with HLHS have a slower rate of global and regional brain growth compared with d-TGA, likely related to inherent physiologic differences postoperatively. These findings demonstrate the complex interplay between cardiac lesion, brain injury, and brain growth. ( $\mathrm{J}$ Thorac Cardiovasc Surg 2018;155:291-300)

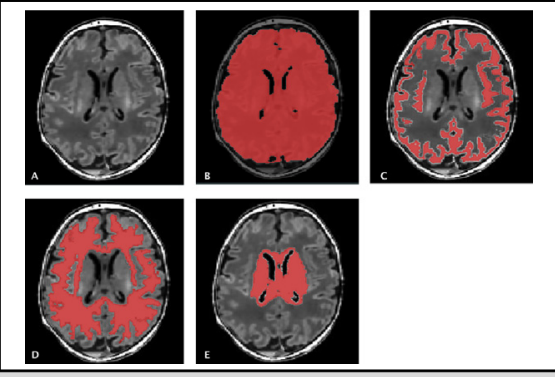

T1-weighted magnetic resonance imaging. A, Automatic segmentation of brain structures to measure (B) total brain; C, Gray matter; D, White matter; and (E) deep gray matter volume.

\section{Central Message}

Neonates with hypoplastic left heart syndrome have a slower rate of perioperative brain growth than d-transposition of the great arteries. Brain injury has less influence on neonatal brain growth.

\section{Perspective}

Brain injury and delayed brain development are common in neonates with hypoplastic left heart syndrome and d-transposition of the great arteries. Despite similarities in brain health preoperatively, neonates with hypoplastic left heart syndrome have a slower rate of ongoing brain growth in the perioperative time period a reflection of the postoperative physiologic differences between these 2 cardiac lesions.

See Editorial Commentary page 301

See Editorial page 289.

\footnotetext{
From the ${ }^{\mathrm{a}}$ Department of Pediatrics, Division of Cardiology, ${ }^{\mathrm{b}}$ Department of Radiology, and ${ }^{c}$ Division of Critical Care, University of California San Francisco, San Francisco; ${ }^{\mathrm{d}}$ Department of Neurology, University of Southern California, Los Angeles, Calif; ${ }^{\mathrm{e}}$ Department of Pediatric Cardiovascular and Thoracic Surgery, University of British Columbia, Vancouver; and ${ }^{\mathrm{f}}$ Department of Neurology, the University of Toronto, Hospital for Sick Children, Toronto, Ontario, Canada. This work was supported by grants K23 NS099422, R01 NS40117, R01NS063876, R01EB009756, R01HD07274, P01 NS082330, and P50 NS35902 from the National Institutes of Health; grant MOP93780 from the Canadian Institutes of Health Research; grant 5-M01-RR-01271 from the National Center for Research Resources; grants 5-FY05-1231 and 6-FY2009-303 from the March of Dimes Foundation; grant 0365018Y from the American Heart Association; and grant 2002/3E
}

from the Larry L. Hillblom Foundation. Dr Miller is the Bloorview Children's Hospital Chair in Pediatric Neuroscience.

Drs Peyvandi and Kim contributed equally to this article.

Received for publication Feb 21, 2017; revisions received July 10, 2017; accepted for publication Aug 5, 2017; available ahead of print Sept 13, 2017.

Address for reprints: Shabnam Peyvandi, MD, UCSF Benioff Children's Hospital, Pediatric Heart Center, 550 16th St, 5th Floor, San Francisco, CA 94158 (E-mail: Shabnam.peyvandi@ucsf.edu).

0022-5223/\$36.00

Copyright (C) 2017 by The American Association for Thoracic Surgery

http://dx.doi.org/10.1016/j.jtcvs.2017.08.019 


\section{Abbreviations and Acronyms}

BIS = brain injury severity

$\mathrm{CHD}=$ congenital heart disease

$\mathrm{GA}=$ gestational age

$\mathrm{GM}=$ gray matter

HLHS = hypoplastic left heart syndrome

IVH = intraventricular hemorrhage

IQR = interquartile range

MRI = magnetic resonance imaging

$\mathrm{TBV}=$ total brain volume

$\mathrm{d}$-TGA $=$ d-transposition of the great arteries

$\mathrm{WM}=$ white matter

$\mathrm{WMI}=$ white matter injury

Scanning this QR code will take you to supplemental figures and appendix for this article.

Advances in surgical techniques and perioperative care have led to improved survival of newborns with critical congenital heart disease (CHD), such as hypoplastic left heart syndrome (HLHS) and d-transposition of the great arteries (d-TGA). ${ }^{1}$ Although there has been a decline in gross neurologic insults in these children, many experience behavioral, emotional, cognitive, and motor impairments, suggesting widespread brain dysfunction. ${ }^{2,3}$ Studies that have assessed neonates with d-TGA and HLHS as a single cohort have identified delayed brain development and a similar prevalence of preoperative brain injury despite the fact that these lesions are anatomically and physiologically distinct from one another. ${ }^{4-7}$ Interestingly, both lesions have evidence of altered fetal cerebral oxygen delivery and smaller total brain volumes (TBVs) as compared with the normal fetus, although the underlying mechanisms may differ in each lesion. ${ }^{8-10}$

Measurements of simple metrics of brain growth on magnetic resonance imaging (MRI) have identified smaller total and regional brain size at birth and infancy in CHD compared with controls ${ }^{11,12}$; however, the rate of brain growth was similar and did not differ by cardiac lesion. ${ }^{11}$ Cerebral MRI volumetry is a quantitative measure of total and regional brain volume providing an accurate means to assess brain volume and growth trajectory. ${ }^{13-15}$ Sophisticated automated volumetry techniques can model brain tissue characteristics during neonatal development and have identified decreased total and regional brain volumes in neonates with complex CHD previous to any corrective operation. ${ }^{16}$ In particular, studies have shown associations between smaller total and regional brain volumes (white matter [WM] and cortical gray matter [GM]) with neurodevelopmental outcome in adolescents with $\mathrm{CHD},{ }^{17}$ as well as association between brain volume at birth and neonatal neurobehavior. ${ }^{18}$ These studies highlight the potential usefulness of this quantitative measure in identifying the patients at greatest risk for neurodevelopmental impairment.

We sought to assess the trajectory of perioperative brain growth in relationship to cardiac diagnosis and acquired brain injuries by using magnetic resonance morphometry in a large sample of well-characterized patients with standardized imaging time points in the neonatal period. We hypothesize that patients with HLHS exhibit slower rates of brain growth due to a palliative, staged surgical approach with persistent cyanosis and potential for ongoing postoperative brain injury. In contrast, newborns with dTGA undergo definitive surgical correction with restoration of normal brain oxygen delivery, providing more favorable conditions for brain growth.

\section{METHODS}

Between 2001 and 2014, newborns with critical CHD at the University of California San Francisco Benioff Children's Hospital were invited consecutively to participate in a prospective protocol studying brain development and brain injury in CHD using MRI. Brain imaging findings from earlier versions of this cohort were reported previously. ${ }^{4,5}$ Patients who were born before 36 weeks' gestation, had a suspected congenital infection, had clinical evidence of a congenital malformation or syndrome, and/or had a suspected or confirmed genetic or chromosomal anomaly were excluded. Once written informed consent was received, patients underwent brain MRI before and after cardiac surgery. The institutional committee on human research approved the study protocol. This study was approved by the UCSF IRB study number 10-03749, original approval date October 2001.

Patients diagnosed as having d-TGA with or without a ventricular septal defect or HLHS were included in this current study. HLHS was defined as the presence of 1 functioning right ventricle with varying degrees of severe left heart hypoplasia requiring a palliative surgical intervention for survival (ie, Stage I operation) in the newborn period. All subjects had a Stage I (Norwood) operation with a RV-PA conduit (Sano) modification.

\section{MRI Study}

Preoperative MRI studies were performed as soon as the baby could be safely transported to the MRI scanner as determined by the clinical team. Postoperative studies were performed after completion of perioperative care and before discharge from the hospital. Imaging time points were separated by an average of 15 days in the entire cohort. Detailed MRI methods are listed in Appendix E1. A neuroradiologist (A.J.B.) reviewed each MRI. Brain injury was characterized as stroke, white matter injury (WMI), intraventricular hemorrhage (IVH), and/or global hypoxic ischemic injury as previously described. ${ }^{5,6}$ Postoperative brain injuries are limited to newly acquired lesions not evident on the preoperative scan. WMI was classified as mild (1-3 foci each $<2 \mathrm{~mm})$, moderate ( $>3$ foci or any foci $>2 \mathrm{~mm}$ ), or severe ( $>5 \%$ of white matter volume). ${ }^{5}$ IVH was characterized as grade I, II, III, or IV via the system of Papile and colleagues. ${ }^{19}$ In addition, brain injury severity (BIS) was determined for each patient as previously described. ${ }^{20}$ The BIS was assigned as follows: 0 indicates normal (no injury); 1, minimal injury (minimal WMI 

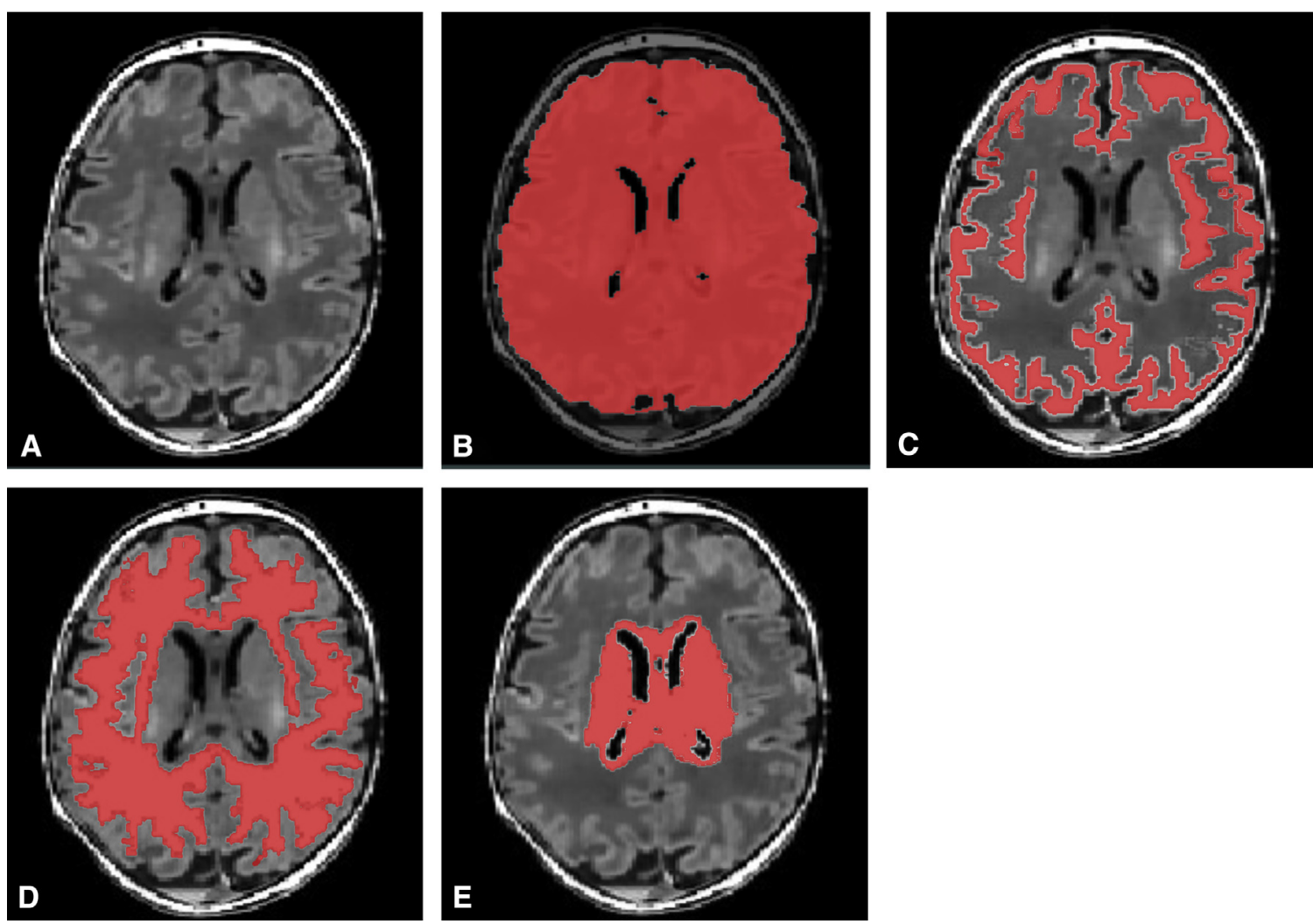

FIGURE 1. T1-weighted magnetic resonance imaging. A, Automatic segmentation of brain structures to measure (B) total brain; C, Gray matter; D, White matter; and (E) deep gray matter volumes.

and IVH grade I or II); 2, stroke (all stroke); and 3, moderate-to-severe injury (moderate and severe WMI, IVH grade III, or global hypoxic-ischemic injury).

\section{Morphometry}

With an automated approach, segmentation of the GM, WM, and deep GM structure (combining the thalamus, the basal ganglia, and the periventricular germinal zone) was performed, as previously described (Figure 1). ${ }^{21}$ Detailed methods are listed in Appendix E1.

\section{Clinical Variables}

Clinical data were collected prospectively from the medical records by a team of trained neonatal research nurses and reviewed by a pediatric intensivist (P.M.) who was blinded to all neuroimaging findings.

\section{Statistical Analysis}

Demographic characteristics, descriptors of brain injury, and clinical variables were compared between patients with HLHS and d-TGA by the use of standard descriptive statistics. To analyze brain structural volumes, we used general linear models that included gestational age (GA) at scan, type of cardiac lesion (d-TGA or HLHS), BIS, and sex as dependent variables, whereas $\mathrm{TBV}, \mathrm{GM}$, WM, or deep GM volumes were set as the independent variables. As most subjects were scanned twice (one before and one after the surgery), mixed-effect linear models were used to take into account multiple measurements per subject. To assess whether preoperative or postoperative brain injury influenced brain volumes, all subjects were first dichotomized into those with a cumulative BIS score $>0$ and those with a BIS score $=0$ based on the maximum value between preoperative and postoperative BIS scores.
In a separate analysis, an interaction term was included as BIS $\times \mathrm{GA}$ at scan into the linear model to assess differences in brain growth rate depending on the presence of brain injury.

Finally, to assess difference by cardiac lesion, a linear model that contained a group term of cardiac lesion type (d-TGA or HLHS) and GA at scan as the main effect term was performed, including an interaction term (cardiac lesion $\times$ GA at scan). Adjustments were made for the presence of brain injury. The Bonferroni adjustment for multiple comparisons was used to obtain corrected $P$ values, which after adjusting for 8 comparisons was $P=.006$

\section{RESULTS}

A total of 79 infants were included in this study, 49 with d-TGA and 30 with HLHS. There was a total of 79 preoperative brain MRIs and 73 postoperative brain MRIs. The postoperative MRI was not analyzed in 5 subjects due to motion degradation. One subject did not have a postoperative MRI due to pacemaker placement. Demographics and clinical variables are listed in Tables 1 and 2. Both groups were similar in preoperative clinical characteristics including birth weight and birth head circumference. Postoperatively, subjects with HLHS had more frequent cardiac arrest events and a longer length of hospital stay compared with subjects with d-TGA. In addition, the postoperative MRI was performed on average 6.5 days later in the HLHS group (day-of-life MRI: median 
TABLE 1. Demographics by cardiac lesion

\begin{tabular}{lccc}
\hline & d-TGA $(\mathbf{n}=\mathbf{4 9})$ & HLHS $(\mathbf{n}=\mathbf{3 0})$ & $P$ value \\
\hline EGA delivery, wk (mean, 95\% CI) & $39.2(38.8-39.6)$ & $38.9(38.4-39.3)$ & .35 \\
Birth weight, g (mean, 95\% CI) & $3396.8(3228.0-3565.6)$ & $3211.9(3030.6-3393.3)$ & $16(53.3)$ \\
Male, n (\%) & $39(79.6)$ & $34.1(33.6-34.6)$ & .16 \\
Birth head circumference, cm (mean, 95\% CI) & $34.0(33.7-34.3)$ & $3308.5(3183.1-3433.8)$ & .78 \\
Weight at MR2, g (mean, 95\% CI) & $3492.9(3364.6-3621.4)$ & .06 \\
\hline
\end{tabular}

$\overline{d-T G A}$, d-Transposition of the great arteries; $H L H S$, hypoplastic left heart syndrome; $E G A$, estimated gestational age; $C I$, confidence interval; $M R 2$, postoperative magnetic resonance imaging.

24 days, interquartile range [IQR]: 20-30) as compared with the d-TGA group (day-of-life MRI: median 17.5 days, IQR: $15-25, P=.001)$. The average time between scans was 15 days (IQR 10-21) in the entire cohort, 12.5 days in d-TGA (IQR 9-17), and 19 days in HLHS (IQR 15-25). Weight at the time of the second MRI was similar in both groups (d-TGA: $3492.9 \mathrm{~g}, 95 \%$ confidence interval, 3364.6-3621.4; HLHS: $3308.5 \mathrm{~g}$; 95\% confidence interval, $3183.1-3433.8 ; P=.06$ ) with a similar increase in weight from birth to the time of the second MRI in both groups (d-TGA: $96.1 \mathrm{~g}$; HLHS: $96.6 \mathrm{~g}$ ) (Table 1).

The frequency of preoperative brain injury was greatest in subjects with HLHS but not statistically significant (dTGA $=14.3 \%$, HLHS $=30 \%, P=.09$ ). In contrast, subjects with HLHS had a greater prevalence of new postoperative injury $(\mathrm{d}-\mathrm{TGA}=30.4 \%$, HLHS $=55.6 \%$, $P=.03$ ), mostly in the form of stroke (Table 3). The cumulative BIS score, which represents the greatest score on either MRI, is represented in Figure 2. Subjects with HLHS had greater cumulative BIS scores than subjects with d-TGA (BIS $=2$ and $3 ; P=.01)$.

\section{Morphometry}

Total and regional brain volumes increased significantly with increasing age in the entire cohort, even when we accounted for the presence of brain injury, sex, and the type of cardiac lesion $(P<.0001$ for all regions). Subjects were dichotomized by cumulative BIS score (BIS $=0$ vs BIS > 0). Overall, mean global and regional brain volumes (all pre- and postoperative data points combined) were greater in subjects without brain injury compared with those with brain injury, except in WM (TBV: $385 \mathrm{~cm}^{3}$ vs $359 \mathrm{~cm}^{3}$, $P<.0001$; GM: $147 \mathrm{~cm}^{3}$ vs $139 \mathrm{~cm}^{3}, P<.0001$; deep GM: $48 \mathrm{~cm}^{3}$ vs $46 \mathrm{~cm}^{3}, P<.004$; WM: $121 \mathrm{~cm}^{3}$ vs $116 \mathrm{~cm}^{3}$, $P=.007)$. Although preoperative brain injury did not predict preoperative brain volumes, it was associated with significantly lower GM volumes on the postoperative MRI $\left(147 \mathrm{~cm}^{3}\right.$ vs $\left.157 \mathrm{~cm}^{3}, P=.0001\right)$. Similarly, postoperative brain injury was associated with lower GM volumes on the postoperative MRI $\left(149 \mathrm{~cm}^{3}\right.$ vs $\left.159 \mathrm{~cm}^{3}, P=.0001\right)$. In contrast, preoperative brain volumes were not associated with new postoperative brain injury $(P=.2)$. Despite the difference in brain volumes at single points in time, the rate of global and regional brain growth did not correlate with the presence of brain injury noted on the pre- or postoperative MRI (rate of growth in those with no brain injury [cumulative BIS $=0$ ] vs those with brain injury [cumulative BIS > 0]; TBV: $12.3 \mathrm{~cm}^{3}$ vs $12.4 \mathrm{~cm}^{3}$, $P>.3$; GM: $8.15 \mathrm{~cm}^{3}$ vs $8.14 \mathrm{~cm}^{3}, P>.5$; WM: $5.52 \mathrm{~cm}^{3}$ vs $5.46 \mathrm{~cm}^{3}, P>.4$; deep GM: $1.21 \mathrm{~cm}^{3}$ vs $1.18 \mathrm{~cm}^{3}$,

TABLE 2. Pre- and postoperative clinical variables by cardiac lesion

\begin{tabular}{|c|c|c|c|}
\hline & d-TGA $(n=49)$ & HLHS $(n=30)$ & $P$ value \\
\hline Balloon atrial septostomy, n (\%) & $30(61.2)$ & $1(3.3)$ & $<.001$ \\
\hline Lowest preoperative $\mathrm{O}_{2}$ sat, mean $(95 \% \mathrm{CI})$ & $56.9(52.0-61.7)$ & $80.1(76.2-84.0)$ & $<.001$ \\
\hline Preoperative pH (first ABG), mean $(95 \%$ CI) & $7.27(7.23-7.31)$ & $7.29(7.24-7.35)$ & .43 \\
\hline Preop base excess (first ABG), mean $(95 \% \mathrm{CI})$ & $-6.2(-8.5$ to -3.9$)$ & $-4.1(-6.7$ to -1.4$)$ & .23 \\
\hline Preoperative cardiac arrest, $\mathrm{n}(\%)$ & $1(2.0)$ & $2(6.7)$ & .55 \\
\hline Day-of-life operation median (IQR) & $8(5.5-11)$ & $8(6-11)$ & .99 \\
\hline Day-of-life MRI 1, median (IQR) & $5(3-6)$ & $5(3-6)$ & .96 \\
\hline Postoperative cardiac arrest, $\mathrm{n}(\%)$ & 0 & $5(16.7)$ & .006 \\
\hline Postoperative ECLS, n (\%) & $2(4.1)$ & $4(13.3)$ & .19 \\
\hline Postoperative duration of mechanical ventilation, median (IQR) & $5(3-7)$ & $8(6-10)$ & .0002 \\
\hline Day-of-life MRI 2, median (IQR) & $17.5(15-25)$ & $24(20-30)$ & .001 \\
\hline Hospital LOS, mean $(95 \% \mathrm{CI})$ & $23.8(19.2-28.3)$ & $40(30.0-50.0)$ & .001 \\
\hline
\end{tabular}

$\overline{d-T G A}$, d-Transposition of the great arteries; $H L H S$, hypoplastic left heart syndrome; $\mathrm{O}_{2}$ sat, oxygen saturation; $C I$, confidence interval; $A B G$, arterial blood gas; $I Q R$, interquartile range; $M R I$ 1, preoperative magnetic resonance imaging; $E C L S$, extracorporeal life support; $M R I 2$, postoperative magnetic resonance imaging; $L O S$, length of stay. 
TABLE 3. Pre- and postoperative brain injury by cardiac lesion

\begin{tabular}{lrrr}
\hline & d-TGA $(\mathbf{n}=\mathbf{4 9})$ & HLHS $(\mathbf{n}=\mathbf{3 0})$ & $\boldsymbol{P}$ value \\
\hline Preoperative & & & \\
Any injury & $17 / 49(34.7 \%)$ & $13 / 30(43.3 \%)$ & .44 \\
WMI & $7 / 49(14.3 \%)$ & $9 / 30(30.0 \%)$ & .09 \\
Stroke & $12 / 49(24.5 \%)$ & $6 / 30(20.0 \%)$ & .64 \\
New postoperative* & & & \\
Any injury & $14 / 46(30.4 \%)$ & $15 / 27(55.6 \%)$ & .03 \\
WMI & $13 / 46(28.3 \%)$ & $9 / 27(33.3 \%)$ & .65 \\
Stroke & $1 / 46(2.2 \%)$ & $7 / 27(25.9 \%)$ & .003 \\
\hline
\end{tabular}

d-TGA, d-Transposition of the great arteries; HLHS, hypoplastic left heart syndrome; $W M I$, white matter injury. *Postoperative magnetic resonance missing in 3 subjects with d-TGA and 3 subjects with HLHS due to death of subject.

$P>.3$; Figure 3, A, Figure E1, A). However, when we only evaluated patients with the most severe form of brain injury (moderate-to-severe WMI, cumulative BIS $=3$ ), the rate of growth in WM and GM was significantly slower compared with those with no brain injury $\left(\mathrm{GM}: 6.7 \mathrm{~cm}^{3} / \mathrm{wk}\right.$ vs $8.1 \mathrm{~cm}^{3} / \mathrm{wk}, P=.001$; WM: $1.2 \mathrm{~cm}^{3} /$ wk vs $1.8 \mathrm{~cm}^{3} / \mathrm{wk}$, $P<.001$; Figure 3, $B$, Figure E1, $B$ ).

When we assessed differences by cardiac lesion, there was no difference in overall mean total and regional measures of brain volume (all pre- and postoperative data points combined and when assessing each time point separately) in subjects with d-TGA compared with HLHS (TBV: $375 \mathrm{~cm}^{3}$ vs $372 \mathrm{~cm}^{3}, P>.2$; GM: $142 \mathrm{~cm}^{3}$ vs
$145 \mathrm{~cm}^{3}, P>.1$; WM: $120 \mathrm{~cm}^{3}$ vs $117 \mathrm{~cm}^{3}, P=.1$; deep GM: $47 \mathrm{~cm}^{3}$ vs $46 \mathrm{~cm}^{3}, P>.1$ ). However, subjects with d-TGA had a faster rate of increase in global and regional brain growth compared with subjects with HLHS, even when we adjusted for GA at scan and the presence of any injury (TBV: $12 \mathrm{~cm}^{3} / \mathrm{wk}$ vs $7 \mathrm{~cm}^{3} / \mathrm{wk}$; WM volume: $2.1 \mathrm{~cm}^{3} / \mathrm{wk}$ vs $0.6 \mathrm{~cm}^{3} / \mathrm{wk}$; deep GM volume: $1.5 \mathrm{~cm}^{3} / \mathrm{wk}$ vs $0.7 \mathrm{~cm}^{3} / \mathrm{wk} ; P<.001$; Figure 4 , Figure E2). GM did not show a significant difference in growth by cardiac lesion $(P=.2)$. Even when we excluded subjects with moderate-to-severe WMI (BIS $=3$ ), those with d-TGA had a faster rate of increase in global and regional brain growth compared with subjects with HLHS $(P<.001$ for TBV, deep GM, and WM).

\section{DISCUSSION}

Our results demonstrate better perioperative brain growth in patients with d-TGA compared with HLHS. Specifically, there was a $40 \%$ increase in the rate of brain growth in WM, GM, and TBV in subjects with d-TGA in this short period from preoperative to postoperative imaging. Interestingly, although subjects with brain injury had overall smaller brain volumes at individual points in time compared with those without injury, the strongest predictor of brain growth trajectory was cardiac lesion. This is the first report, to our knowledge, directly comparing the perioperative patterns of brain growth between 2 infant groups with common, yet
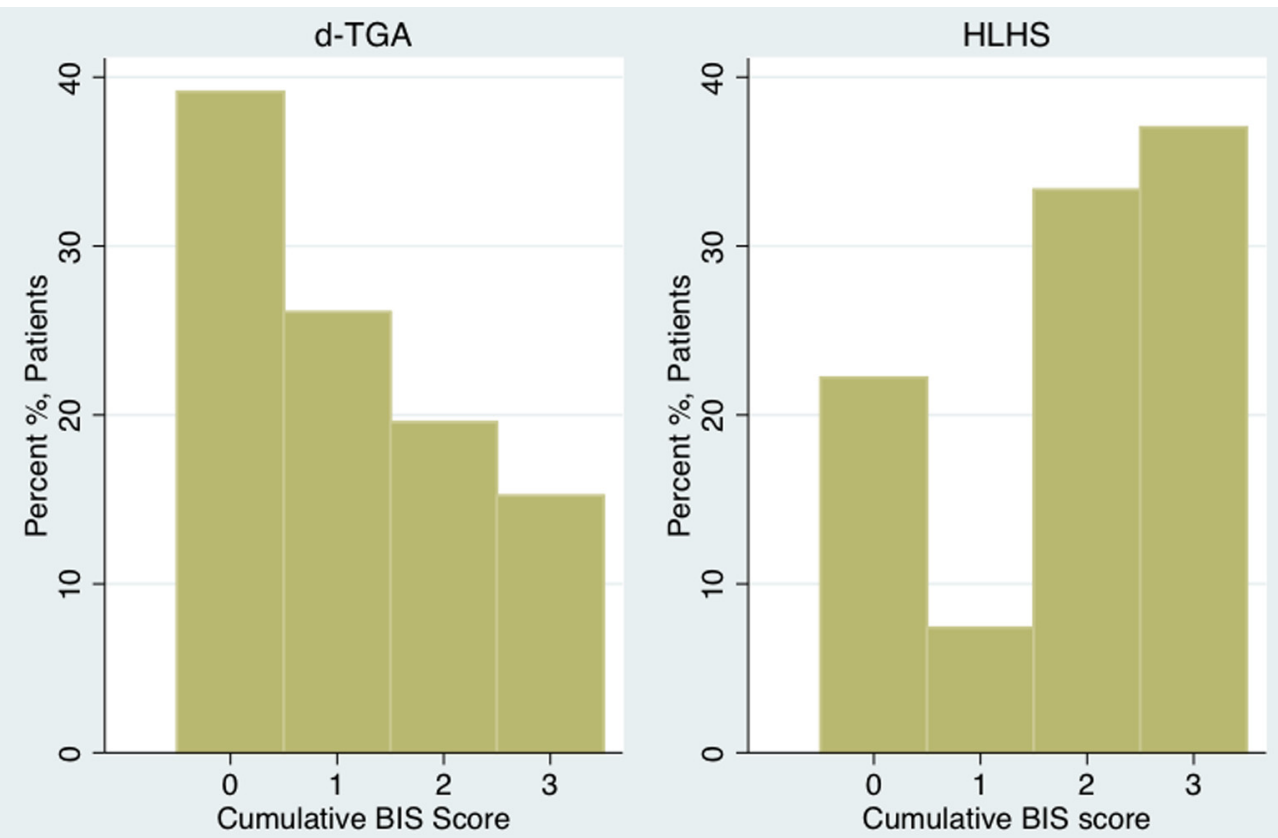

Graphs by cardiacgrp

FIGURE 2. Distribution of the cumulative BIS score by cardiac lesion. The cumulative BIS score is the worst score when combining the pre- and postoperative magnetic resonance imaging. A test for trends demonstrates less-severe cumulative BIS scores in the d-TGA group $(P=.01)$. $d$-TGA, d-transposition of the great arteries; HLHS, hypoplastic left heart syndrome; BIS, brain injury severity. 


\section{Comparison between babies with $\mathrm{BIS}=0$ and those with $\mathrm{BIS}=1-3$}
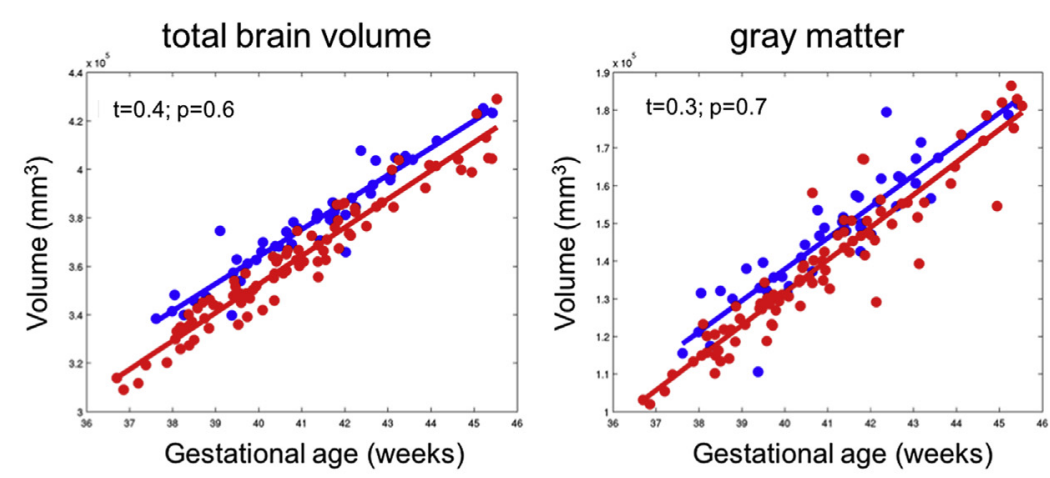

$$
\begin{aligned}
& -\mathrm{BIS}=0 \\
& -\mathrm{BIS}=1-3
\end{aligned}
$$

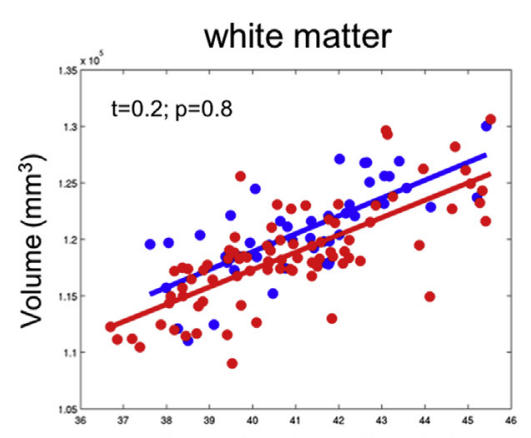

Gestational age (weeks)

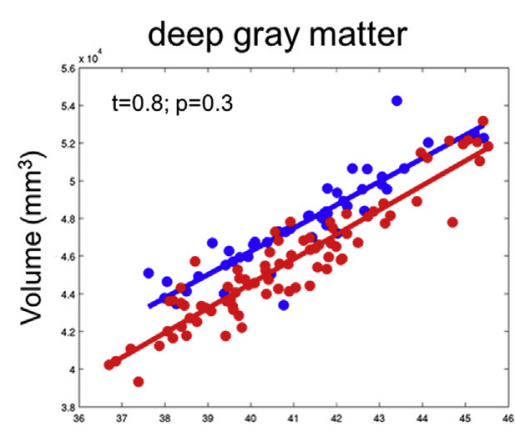

Gestational age (weeks)

\section{A}

\section{Comparison between babies with $\mathrm{BIS}=0$ and those with $\mathrm{BIS}=3$}
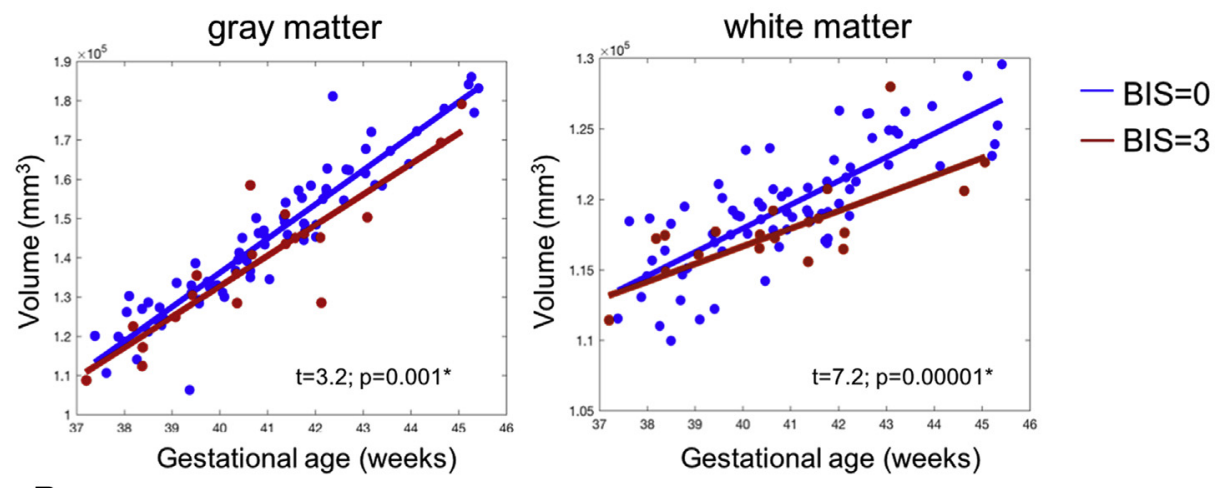

B

FIGURE 3. A, Brain global/regional volume changes in the presence of brain injury (red line, cumulative BIS $>0$ ) and without brain injury (blue line, cumulative BIS $=0$ ). The $\mathrm{x}$-axis represents the gestational age at the time of MRI, and the $\mathrm{y}$-axis represents the volume in $\mathrm{mm}^{3}$. The plot includes preand postoperative brain MRI measures for each subject with a best-fitted line. Plots with paired observations for each subject are included in Figure E1. Overall, mean global and regional (gray matter and deep gray matter) brain volumes were lower in subjects with brain injury compared with those without brain injury. However, the rate of global and regional volume change over time was not associated with brain injury (all $P$ values $>.1$ ). B, Brain regional volume changes in gray and white matter in the presence of no brain injury (blue line, cumulative BIS $=0$ ) and in those with moderate-to-severe white matter injury (dark red line, cumulative BIS $=3$ ). Those with moderate-to-severe white matter injury have significantly slower growth in gray matter and white matter as compared with those without injury $(P=.001$ and $P<.0001$, respectively). BIS, Brain injury severity.

physiologically distinct critical congenital cardiac defects using advanced magnetic resonance morphometry.

In the fetal time period, both d-TGA and HLHS fetuses have evidence of delayed brain development (TBV and metabolic brain development), ${ }^{9}$ although the underlying mechanisms likely differ by cardiac physiology and anatomy. In particular, based on fetal lamb models, those with HLHS have abnormalities in both perfusion (due to 

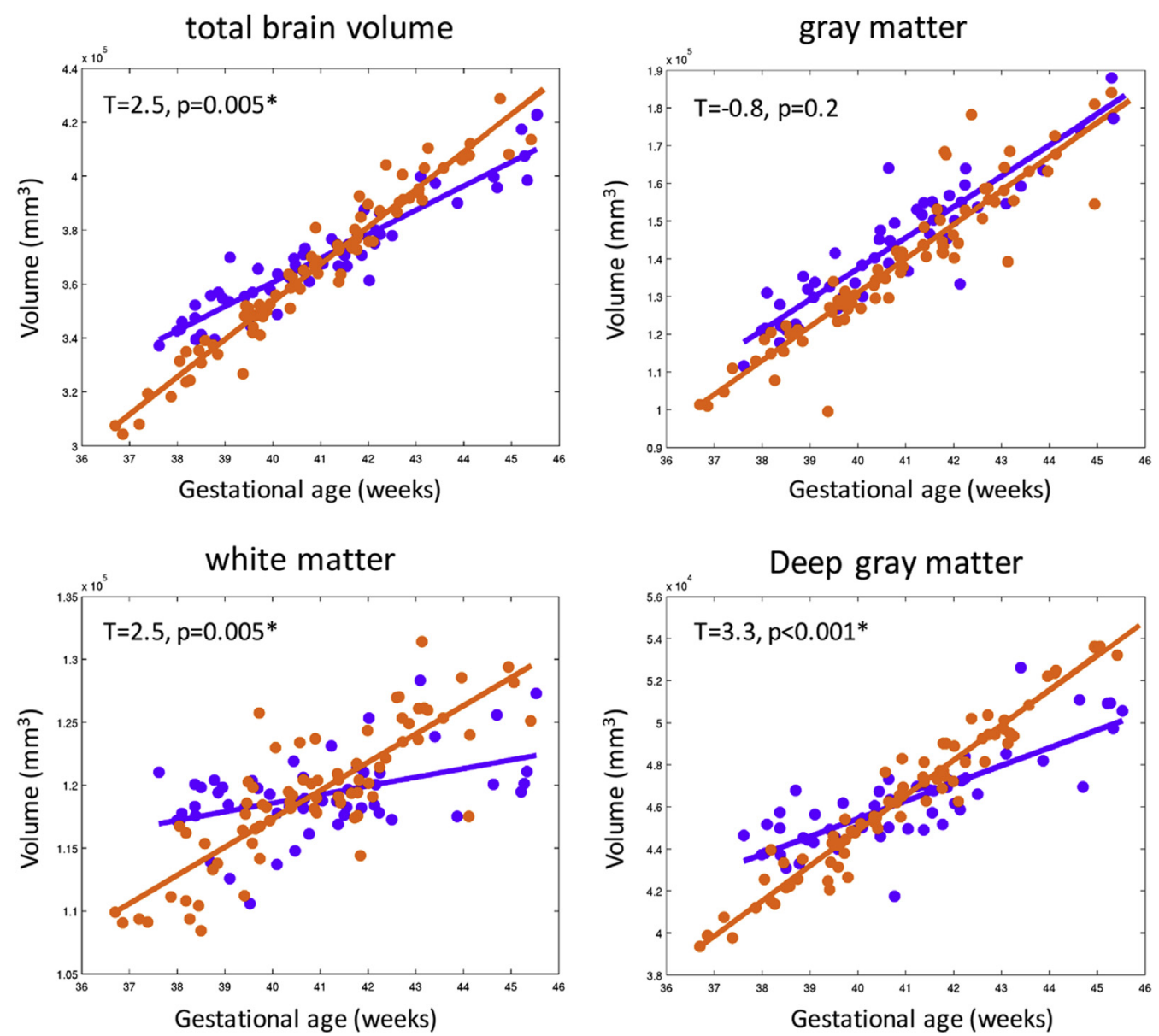

FIGURE 4. The rate of change in global and regional brain volumes by cardiac lesion after adjusting for the presence of brain injury. The $x$-axis represents the gestational age at the time of MRI, and the $y$-axis represents the volume in $\mathrm{mm}^{3}$. The plots include pre- and postoperative brain MRI measures for each subject with a best-fitted line. Plots with paired observations for each subject are included in Figure E2. Subjects with HLHS (purple line) have a slower rate of growth in total brain volume, white matter, and deep gray matter as compared with those with d-TGA (orange line). *Significant after Bonferroni correction, $P<.006$.

retrograde flow from the ductus arteriosus) and oxygenation of cerebral blood flow. In contrast, those with d-TGA are more likely to have abnormalities in oxygenation of cerebral blood flow. ${ }^{10}$ Ultimately, both lesions result in decreased cerebral oxygen delivery and consumption. ${ }^{8}$ Recently, Rudolph ${ }^{22}$ suggested that decreased substrate delivery to the brain such as glucose may be the primary cause for the abnormalities in brain development. Most studies find no differences by cardiac lesion in the degree of fetal or neonatal preoperative brain development. ${ }^{4,7}$ Some evidence suggests that within a particular group, specific anatomic features such as aortic atresia in HLHS lead to the greatest degree of delayed microstructural brain development. ${ }^{23}$ Our study did not demonstrate significant differences preoperatively in measures of brain volumes when we compared HLHS with d-TGA. This demonstrates that although these lesions are physiologically different, brain growth is similar during fetal and up to the preoperative time period.
After birth and before their neonatal operation, patients with both d-TGA and HLHS are at risk for brain injury in the form of WMI and stroke. ${ }^{5,24,25}$ Indeed, both groups have a similar risk of brain injury, likely secondary to shared risk factors such as hypoxia, length of time to surgery, embolism, and preoperative cardiac arrest. ${ }^{26-29}$ New, postoperative brain injury is common and tends to be more prevalent in subjects with single-ventricle physiology. ${ }^{5}$ Similarly, we found that postoperative brain injury was more common in the HLHS group, with a greater number of small focal strokes, consistent with embolism. As opposed to patients with d-TGA, patients with HLHS after a Norwood procedure are vulnerable to embolic injury, which may be modifiable based on postoperative management and hospital length of stay.

The relationship between brain development and injury is complex and dependent on methods used to measure brain development. Several studies agree that brain immaturity, measured by the use of semiquantitative methods, is a risk 
factor for preoperative brain injury. ${ }^{20,28,30}$ However, our measures of total and regional brain volumes preoperatively did not seem to predict the presence of new postoperative brain injury. The impact of brain injury on measures of ongoing brain development is less clear. For example, our group has shown that preoperative brain injury predicts delayed postoperative metabolic and microstructural brain development. ${ }^{20} \mathrm{We}$ found that preoperative brain injury is associated with smaller postoperative GM volumes and that overall brain volumes were smaller in those with injury compared with those without injury. However, we found that rate of brain growth did not differ by the presence of brain injury globally or regionally. Thus, although brain injury is associated with brain volume loss, there appears to be minimal influence on continued brain growth, at least in the perioperative time period. On the most severe end of the spectrum, subjects with moderate-to-severe WMI exhibit a slower rate of growth in WM and GM. We believe this result is driven by the complex interplay between cardiac lesion, brain injury, and brain growth. In our cohort, a larger percentage of subjects with HLHS had moderate-to-severe WMI, suggesting that cardiac lesion is the initial risk factor that influences both injury and brain growth. In fact, when subjects with moderate-to-severe WMI were removed from the analysis, those with d-TGA continued to have an even faster rate of global and regional brain growth as compared with HLHS.

Studies that have analyzed pyramidal tract maturation in newborns with CHD have demonstrated less rapid changes in fractional anisotropy over the perioperative time period in subjects with preoperative brain injury, ${ }^{31}$ similar to what is seen in premature infants with brain injury. ${ }^{32}$ This suggests that the effects of brain injury on brain development may differ at macroscopic and microscopic scales. The techniques used to measure these different aspects of brain development are unique and offer different perspectives on brain maturity. More sensitive measures of brain growth in regions corresponding to the location of acquired focal lesions may lead to a deeper understanding of the longer term effects of milder forms of brain injury on brain growth.

The relationship between brain volumes in particular and injury is inherently complicated. Brain injury may lead to swelling and increased volume acutely, followed by tissue loss, potential repair, and finally, in the immature brain, variable subsequent growth. Brain growth is one component of several factors that may impact long-term neurologic outcomes in patients with complex CHD. The experience with preterm infants has demonstrated the close interplay between injury and continued brain growth and their collective influence on outcomes. Specifically, studies in premature infants have demonstrated long-term effects of WMI or periventricular leukomalacia on brain growth and function. ${ }^{33}$ Although periventricular leukomalacia is localized to the WM, it can lead to disruption of cortical activity ${ }^{34}$ and GM hypoplasia, ${ }^{35}$ with important consequences for developing cortical circuits. ${ }^{36-39}$ Neuropathologic studies in nonsurvivors of critical CHD have shown abnormalities in the thalamus with thalamic neuronal loss and gliosis, ${ }^{40}$ regions critical for working memory and attention, a common deficit noted in survivors of critical CHD. Our data demonstrate that moderate-to-severe WMI does appear to slow the rate of brain growth in WM and GM. Although this is likely largely driven by cardiac lesion, the impact of injury on brain growth and long-term neurodevelopmental outcomes warrants further study.

Our findings demonstrate a clear link between cardiac subgroup and ongoing perioperative brain growth. Given the high burden of acquired injury and delayed brain development early in life, the potential for repair and optimal brain growth is critical. Although patients with HLHS and dTGA appear to share similar fetal and preoperative risk factors, they differ significantly postoperatively. Subjects with d-TGA undergo a corrective operation (arterial switch operation) that restores normal cardiovascular physiology, whereas those with HLHS undergo a series of palliative operations, never achieving normal circulation, with ongoing hypoxia and risk for diminished systemic perfusion. Patients with single-ventricle physiology rely on circulation in series that may result in diminished systemic perfusion with compromised overall cerebral blood flow. There are several other differences between these groups, including operative strategies (low- or full-flow bypass only vs circulatory arrest or regional cerebral perfusion) and postoperative management (length of stay, duration of mechanical ventilation) that influence our findings. Finally, somatic growth likely plays a role in brain development, particularly for those with single-ventricle physiology. However, in the short time period evaluated in our study, both groups had a similar rate of weight gain in the perioperative time period. In line with the cardiac physiology influences on brain development in $\mathrm{CHD}$, we recently reported a relationship between the benefits of prenatal diagnosis and a healthier preoperative state with perioperative brain development. ${ }^{41}$

Ibuki and colleagues ${ }^{42}$ demonstrated normalization of total and frontal brain volumes at 3 years of age in subjects with d-TGA but not in single-ventricle patients. Their data suggested an association between hypoxia (oxygen saturation) and measures of neurodevelopmental outcome (psychomotor development index on the Bayley Scales of Infant and Toddler Development, Second Edition) in the single-ventricle group. Their study was limited due to a small sample size, considerable variability, and long intervals between imaging studies. In our study, the larger sample size, standardized imaging timepoints in the 
neonatal perioperative period, and the short interval between MRI studies strengthens our conclusions and suggests that the differences in brain growth by cardiac lesion begin soon after the neonatal operation.

Our findings suggest improved perioperative brain growth in subjects with d-TGA; however, evidence suggests that these patients continue to have structural and functional neurologic abnormalities later in life. Adolescents with d-TGA have altered GM volume and thickness ${ }^{43}$ as well as diminished WM microstructure ${ }^{17,44,45}$ compared with healthy controls. Neurodevelopmental assessments have revealed ongoing impairments in executive skills, visual-spatial skills, and the need for remedial services, ${ }^{46}$ suggesting that abnormal fetal and preoperative physiology and acquired brain injury may have lasting effects. Indeed, patients with HLHS demonstrate the greatest deficits and neurodevelopmental morbidity. ${ }^{2,47-49}$

Our findings are limited by not knowing the exact timing of acquired brain injury despite 2 imaging time points in the perioperative time period. Although we are invoking developmental etiologies (lack of oxygen and substrate delivery) as the primary influence on neonatal brain growth, the impact of injury as a destructive etiology on brain growth requires a larger sample size and more imaging timepoints, particularly in the transitional period after birth.

In conclusion, brain growth in the perioperative time period is influenced more by cardiac subgroup than by brain injury. Subjects with HLHS have a slower rate of perioperative brain growth globally and regionally in WM and deep GM. Further studies are needed to determine the predictive abilities of this measure of brain development on long-term neurodevelopmental outcomes, with a goal of identifying specific impairments as targets for early intervention.

\section{Conflicts of Interest Statement}

Authors have nothing to disclose with regard to commercial support.

\section{References}

1. Karamlou T, Diggs BS, Ungerleider RM, Welke KF. Evolution of treatment options and outcomes for hypoplastic left heart syndrome over an 18-year period. J Thorac Cardiovasc Surg. 2010;139:119-26; discussion 126-7.

2. Newburger JW, Sleeper LA, Bellinger DC, Goldberg C, Tabbutt S, Lu M, et al. Early developmental outcome in children with hypoplastic left heart syndrome and related anomalies: the single ventricle reconstruction trial. Circulation. 2012;125:2081-91.

3. Bellinger DC, Newburger JW, Wypij D, Kuban KCK, duPlesssis AJ, Rappaport LA. Behaviour at eight years in children with surgically corrected transposition: the Boston Circulatory Arrest Trial. Cardiol Young. 2009;19:86-97.

4. Miller SP, McQuillen PS, Hamrick S, Xu D, Glidden D, Charlton N, et al. Abnormal brain development in newborns with congenital heart disease. $N$ Engl J Med. 2007;357:1928-38.

5. McQuillen PS, Barkovich AJ, Hamrick SEG, Perez M, Ward P, Glidden DV, et al. Temporal and anatomic risk profile of brain injury with neonatal repair of congenital heart defects. Stroke. 2007;38(2 suppl):736-41.
6. Block AJ, McQuillen PS, Chau V, Glass H, Poskitt KJ, Barkovich AJ, et al. Clinically silent preoperative brain injuries do not worsen with surgery in neonates with congenital heart disease. J Thorac Cardiovasc Surg. 2010;140:550-7.

7. Licht DJ, Shera DM, Clancy RR, Wernovsky G, Montenegro LM, Nicolson SC, et al. Brain maturation is delayed in infants with complex congenital heart defects. J Thorac Cardiovasc Surg. 2009;137:529-36; discussion 536-7.

8. Sun L, Macgowan CK, Sled JG, Yoo SJ, Manlhiot C, Porayette P, et al. Reduced fetal cerebral oxygen consumption is associated with smaller brain size in fetuses with congenital heart disease. Circulation. 2015;131:1313-23.

9. Limperopoulos C, Tworetzky W, McElhinney DB, Newburger JW, Brown D, Robertson RL, et al. Brain volume and metabolism in fetuses with congenita heart disease: evaluation with quantitative magnetic resonance imaging and spectroscopy. Circulation. 2010;121:26-33.

10. Rudolph A. Congenital Diseases of the Heart. New York: John Wiley \& Sons: 2011

11. Ortinau C, Inder T, Lambeth J, Wallendorf M, Finucane K, Beca J. Congenital heart disease affects cerebral size but not brain growth. Pediatr Cardiol. 2012 $33: 1138-46$

12. Ortinau C, Beca J, Lambeth J, Ferdman B, Alexopoulos D, Shimony JS, et al Regional alterations in cerebral growth exist preoperatively in infants with congenital heart disease. J Thorac Cardiovasc Surg. 2012;143:1264-70.

13. Gholipour A, Estroff JA, Barnewolt CE, Connolly SA, Warfield SK. Fetal brain volumetry through MRI volumetric reconstruction and segmentation. Int $J$ Comput Assist Radiol Surg. 2011;6:329-39.

14. Matsuzawa J, Matsui M, Konishi T, Noguchi K, Gur RC, Bilker W, et al. Agerelated volumetric changes of brain gray and white matter in healthy infants and children. Cereb Cortex. 2001;11:335-42.

15. Weisenfeld NI, Warfield SK. Automatic segmentation of newborn brain MRI Neuroimage. 2009; 47:564-72.

16. Rhein von M, Buchmann A, Hagmann C, Dave H, Bernet V, Scheer I, et al. Severe congenital heart defects are associated with global reduction of neonata brain volumes. J Pediatr. 2015;167:1259-63.e1.

17. Rhein von M, Buchmann A, Hagmann C, Huber R, Klaver P, Knirsch W, et al Brain volumes predict neurodevelopment in adolescents after surgery for congenital heart disease. Brain. 2014;137:268-76.

18. Owen M, Shevell M, Donofrio M, Majnemer A, McCarter R, Vezina G, et al Brain volume and neurobehavior in newborns with complex congenital heart defects. J Pediatr. 2014;164:1121-7.e1121.

19. Papile LA, Burstein J, Burstein R, Koffler H. Incidence and evolution of subependymal and intraventricular hemorrhage: a study of infants with birth weights less than 1,500 gm. J Pediatr. 1978;92:529-34.

20. Dimitropoulos A, McQuillen PS, Sethi V, Moosa A, Chau V, Xu D, et al. Brain injury and development in newborns with critical congenital heart disease. Neurology. 2013;81:241-8.

21. Kim H, Lepage C, Maheshwary R, Jeon S, Evans AC, Hess CP, et al. NEOCI VET: Towards accurate morphometry of neonatal gyrification and clinical applications in preterm newborns. Neuroimage. 2016;138:28-42.

22. Rudolph AM. Impaired cerebral development in fetuses with congenital cardiovascular malformations: is it the result of inadequate glucose supply? Pediatr Res. 2016;80:172-7.

23. Sethi V, Tabbutt S, Dimitropoulos A, Harris KC, Chau V, Poskitt K, et al. Singleventricle anatomy predicts delayed microstructural brain development. Pediat Res. 2013;73:661-7.

24. Licht DJ, Wang J, Silvestre DW, Nicolosn SC, Montenegro LM, Wernovsky G, et al. Preoperative cerebral blood flow is diminished in neonates with severe congenital heart defects. J Thorac Cardiovasc Surg. 2004;128:841-9.

25. Mahle WT, Tavani F, Zimmerman RA, Nicolson SC, Galli K, Gaynor JG, et al An MRI study of neurological injury before and after congenital heart surgery. Circulation. 2002;106(12 suppl 1):I109-14.

26. McQuillen PS, Hamrick SEG, Perez MJ, Barkovich AJ, Glidden DV, Karl TR, et al. Balloon atrial septostomy is associated with preoperative stroke in neonates with transposition of the great arteries. Circulation. 2006;113:280-5.

27. Petit CJ, Rome JJ, Wernovsky G, Mason SE, Shera DM, Nicolson SC, et al. Preoperative brain injury in transposition of the great arteries is associated with oxygenation and time to surgery, not balloon atrial septostomy. Circulation. 2009;119:709-16.

28. Goff DA, Shera DM, Tang S, Lavin NA, Durning SM, Nicolson SC, et al. Risk factors for preoperative periventricular leukomalacia in term neonates with hypoplastic left heart syndrome are patient related. J Thorac Cardiovasc Surg. 2014; $147: 1312-8$. 
29. Lynch JM, Buckley EM, Schwab PJ, McCarthy AL, Winters ME, Busch DR, et al. Time to surgery and preoperative cerebral hemodynamics predict postoperative white matter injury in neonates with hypoplastic left heart syndrome. J Thorac Cardiovasc Surg. 2014;148:2181-8.

30. Andropoulos DB, Hunter JV, Nelson DP, Stayer SA, Stark AR, McKenzie ED, et al. Brain immaturity is associated with brain injury before and after neonatal cardiac surgery with high-flow bypass and cerebral oxygenation monitoring. $J$ Thorac Cardiovasc Surg. 2010;139:543-56.

31. Partridge SC, Vigneron DB, Charlton NN, Berman JI, Henry RG, Mukherjee P, et al. Pyramidal tract maturation after brain injury in newborns with heart disease. Ann Neurol. 2006;59:640-51.

32. Miller SP, Ferriero DM. From selective vulnerability to connectivity: insights from newborn brain imaging. Trends Neurosci. 2009;32:496-505.

33. Back SA, Miller SP. Brain injury in premature neonates: a primary cerebral dysmaturation disorder? Ann Neurol. 2014;75:469-86.

34. McQuillen PS, Ferriero DM. Perinatal subplate neuron injury: implications for cortical development and plasticity. Brain Pathol. 2005;15:250-60.

35. Inder TE, Huppi PS, Warfield S, Kikinis R, Zientara GP, Barnes PD, et al. Periventricular white matter injury in the premature infant is followed by reduced cerebral cortical gray matter volume at term. Ann Neurol. 1999;46:755-60.

36. Inder TE, Warfield SK, Wang H, Hüppi PS, Volpe JJ. Abnormal cerebral structure is present at term in premature infants. Pediatrics. 2005;115:286-94.

37. Volpe JJ. Encephalopathy of congenital heart disease- destructive and developmental effects intertwined. J Pediatr. 2014;164:962-5.

38. Duerden EG, Guo T, Dodbiba L, Chakravarty MM, Chau V, Poskitt KJ, et al. Midazolam dose correlates with abnormal hippocampal growth and neurodevelopmental outcome in preterm infants. Ann Neurol. 2016;79:548-59.

39. Tam EWY, Miller SP, Studholme C, Chau V, Glidden DV, Poskitt KJ, et al. Differential effects of intraventricular hemorrhage and white matter injury on preterm cerebellar growth. J Pediatr. 2011;158:366-71.

40. Kinney HC, Panigrahy A, Newburger JW, Jonas RA, Sleeper LA. Hypoxic-ischemic brain injury in infants with congenital heart disease dying after cardiac surgery. Acta Neuropathol. 2005;110:563-78.

41. Peyvandi S, De Santiago V, Chakkarapani E, Chau V, Campbell A, Poskitt KJ, et al. Association of prenatal diagnosis of critical congenital heart disease with postnatal brain development and the risk of brain injury. JAMA Pediatr. 2016; 170:e154450.

42. Ibuki K, Watanabe K, Yoshimura N, Kakimoto T, Matsui M, Yoshida T, et al. The improvement of hypoxia correlates with neuroanatomic and developmental outcomes: comparison of midterm outcomes in infants with transposition of the great arteries or single-ventricle physiology. J Thorac Cardiovasc Surg. 2012; $143: 1077-85$

43. Watson CG, Asaro LA, Wypij D, Robertson RL, Newburger JW, Rivkin MJ Altered gray matter in adolescents with d-transposition of the great arteries. J Pediatr. 2016;169:36-43.e1.

44. Rivkin MJ, Watson CG, Scoppettuolo LA, Wypij D, Vajapeyam S, Bellinger DC, et al. Adolescents with D-transposition of the great arteries repaired in early infancy demonstrate reduced white matter microstructure associated with clinical risk factors. J Thorac Cardiovasc Surg. 2013;146: 543-9.e1.

45. Rollins CK, Watson CG, Asaro LA, Wypij D, Vajapeyam S, Bellinger D, et al. White matter microstructure and cognition in adolescents with congenital heart disease. J Pediatr. 2014;165:936-44. e1-2.

46. Bellinger DC, Wypij D, Rivkin MJ, DeMaso DR, Robertson RL, DunbarMasterson C, et al. Adolescents with d-transposition of the great arteries corrected with the arterial switch procedure: neuropsychological assessment and structural brain imaging. Circulation. 2011;124:1361-9.

47. Marelli A, Miller SP, Marino BS, Jefferson AL, Newburger JW. Brain in congenital heart disease across the lifespan: the cumulative burden of injury. Circulation. 2016;133:1951-62.

48. Wernovsky G. Current insights regarding neurological and developmental abnormalities in children and young adults with complex congenital cardiac disease. Cardiol Young. 2006;16(suppl 1[S1]):92-104.

49. Wernovsky G. Outcomes regarding the central nervous system in children with complex congenital cardiac malformations. Cardiol Young. 2005;15(suppl 1): $132-3$

Key Words: congenital heart disease, neurodevelopment, brain injury, brain development, physiology 


\section{APPENDIX E1. DETAILED MAGNETIC RESONANCE IMAGING (MRI) METHODS}

MRI studies were performed with pharmacologic sedation, as needed, on a 1.5- or 3-Tesla system (GE Healthcare Signa Echo-speed; Waukesha, Wis) with GE EXCITE 1.5T/3T software and included 4-mm-thickness T1-weighted sagittal and axial spin echo, 4-mm-thickness dual-echo T2-weighted spin echo, 1.5-mm-thickness coronal or sagittal volumetric 3-dimensional gradient echo with radiofrequency spoiling images, and a diffusion tensor sequence (repetition time: 7000 milliseconds; echo time: 99.5 milliseconds; 3 -mm section thickness; no gap; 3 repetitions per image, with $18 \times 36 \mathrm{~cm}$ field of view and $128 \times 256$ acquisition matrix), acquiring axial images through the whole brain with an in-plane resolution of $1.4 \times 1.4 \mathrm{~mm}^{2}$. Seven images per axial section were acquired, including a T2-weighted reference image $\left(b=0 \mathrm{~s} / \mathrm{mm}^{2}\right)$ and 6-15 diffusionweighted images $\left(b=700 \mathrm{~s} / \mathrm{mm}^{2}\right)$ in noncollinear gradient directions.

\section{MORPHOMETRY}

T1-weighted MRI underwent intensity nonuniformity correction with $\mathrm{N} 3{ }^{\mathrm{E} 1}$ These images were registered linearly to the spatiotemporal neonatal brain template from the Biomedical Image Analysis Group, Imperial College London (http://biomedic.doc.ic.ac.uk/brain-development/ index.php? $\mathrm{n}=$ Main.Neonatal2). The individual images were first registered to the closest age template and further registered to the oldest brain template using the transformation matrix of the registration between the selected age template and the oldest template. We segmented the cerebrum and cerebellum using a patch-based brain extraction algorithm, ${ }^{\mathrm{E} 2}$ which subsequently were used to calculate the total brain volume once each mask was registered back to the native space where the MRI was acquired using the previously obtained transformation matrix. MRI intensities were recorrected with N3 and normalized within the brain mask. The gray matter (GM), white matter, and cerebrospinal fluid were classified within the mask via a recently developed method ${ }^{\mathrm{E} 3}$ that integrated the nonlocal mean estimation of multiatlas texture patches with an advanced label fusion based on a joint probability between selected templates. ${ }^{\mathrm{E} 4}$ To overcome poor segmentation due to varying tissue contrast and varying cortical folding pattern during the postnatal period as well as heavy motion artifact, we constructed a patch library that contains individual atlases (image + manual segmentation; $\mathrm{n}=35$ ) scanned at various ages and under variable conditions of motion. The same segmentation method was used to segment deep GM and ventricles independently. Finally, we measured volumes of GM, white matter, and deep GM by summing the voxel-wise partial volume measurement for each structure, which was given as the posterior probability map resulting from the segmentation algorithm.

\section{E-References}

E1. Sled JG, Pike GB. Standing-wave and RF penetration artifacts caused by elliptic geometry: an electrodynamic analysis of MRI. IEEE Trans Med Imaging. 1998; 17:653-62.

E2. Eskildsen SF, Coupé P, Fonov V, Manjón JV, Leung KK, Guizard N, et al BEaST: brain extraction based on nonlocal segmentation technique. Neuroimage. 2012;59:2362-73.

E3. Kim H, Lepage C, Maheshwary R, Jeon S, Evans AC, Hess CP, et al. NEOCIVET: Towards accurate morphometry of neonatal gyrification and clinical applications in preterm newborns. Neuroimage. 2016;138:28-42.

E4. Coupé P, Manjón JV, Fonov V, Pruessner J, Robles M, Collins DL. Patch-based segmentation using expert priors: application to hippocampus and ventricle segmentation. Neuroimage. 2011;54:940-54. 


\section{Comparison between babies with $\mathrm{BIS}=0$ and those with $\mathrm{BIS}=1-3$}
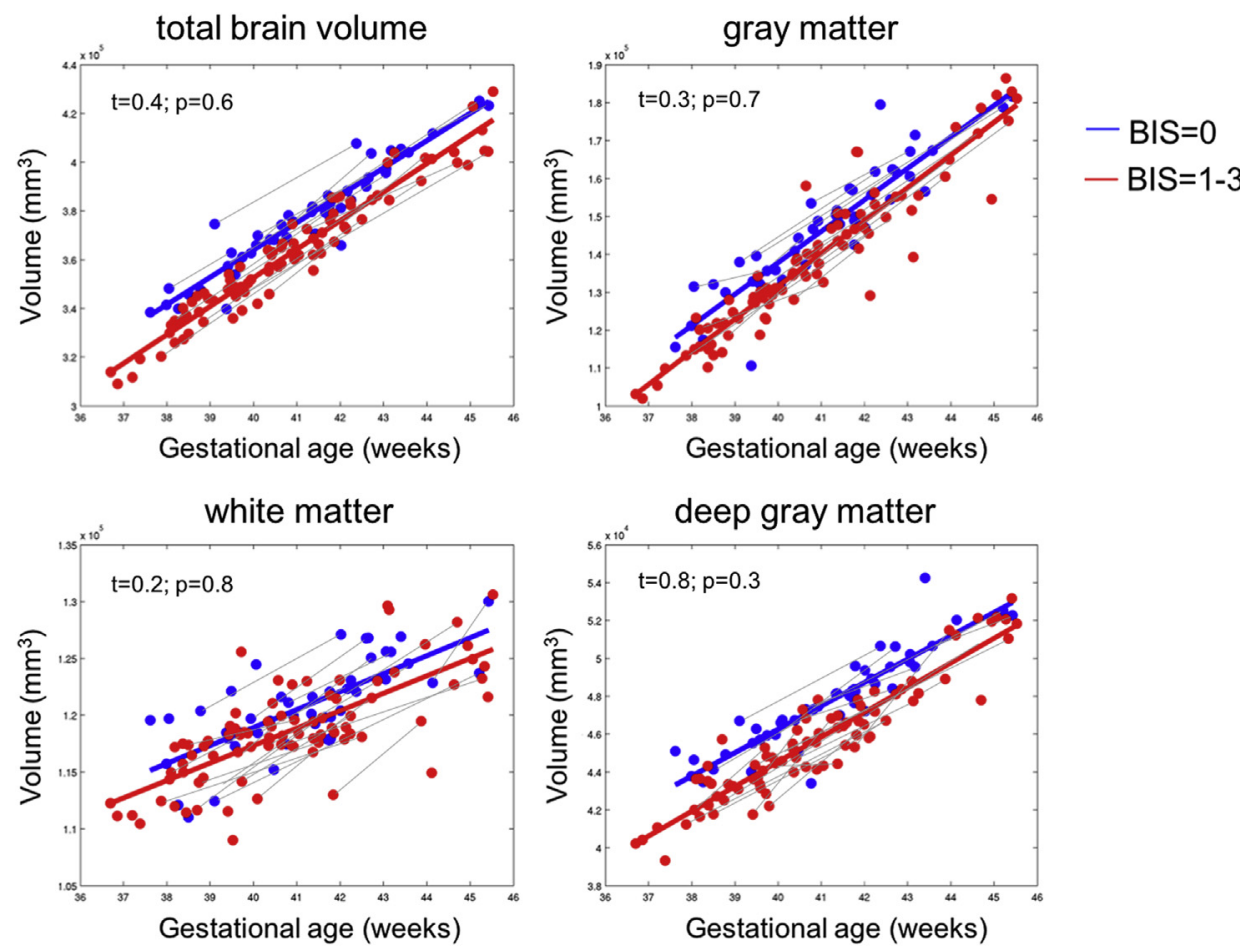

A

\section{Comparison between babies with $\mathrm{BIS}=0$ and those with $\mathrm{BIS}=3$}
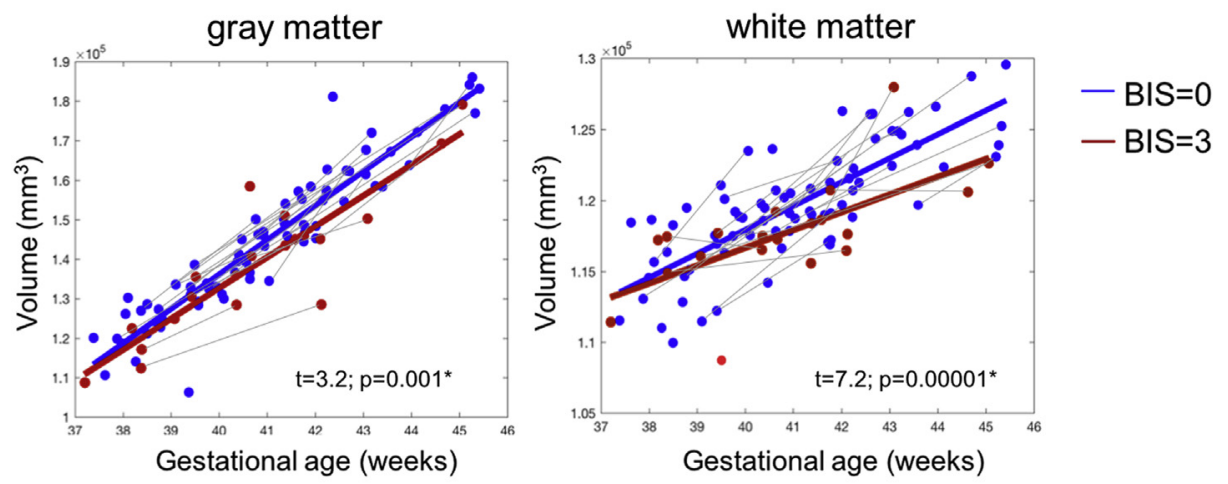

B

FIGURE E1. A, Brain global/regional volume changes in the presence of brain injury (red line, cumulative BIS $>0$ ) and without brain injury (blue line, cumulative BIS $=0$ ). The $\mathrm{x}$-axis represents the gestational age at the time of MRI, and the y-axis represents the volume in $\mathrm{mm}^{3}$. The plot includes pre- and postoperative brain MRI measures with paired observations for each subject (gray line) and a best-fit line. The rate of global and regional volume change over time was not associated with brain injury (all $P$ values $>.1$ ). B, Brain regional volume changes in gray and white matter in the presence of no brain injury (blue line, cumulative BIS $=0$ ) and in those with moderate-to-severe white matter injury (dark red line, cumulative BIS $=3$ ). Those with moderate-tosevere WMI have significantly slower growth in gray matter and white matter compared with those without injury $(P=.001$ and $P<.0001$, respectively). $B I S$, Brain injury severity. 

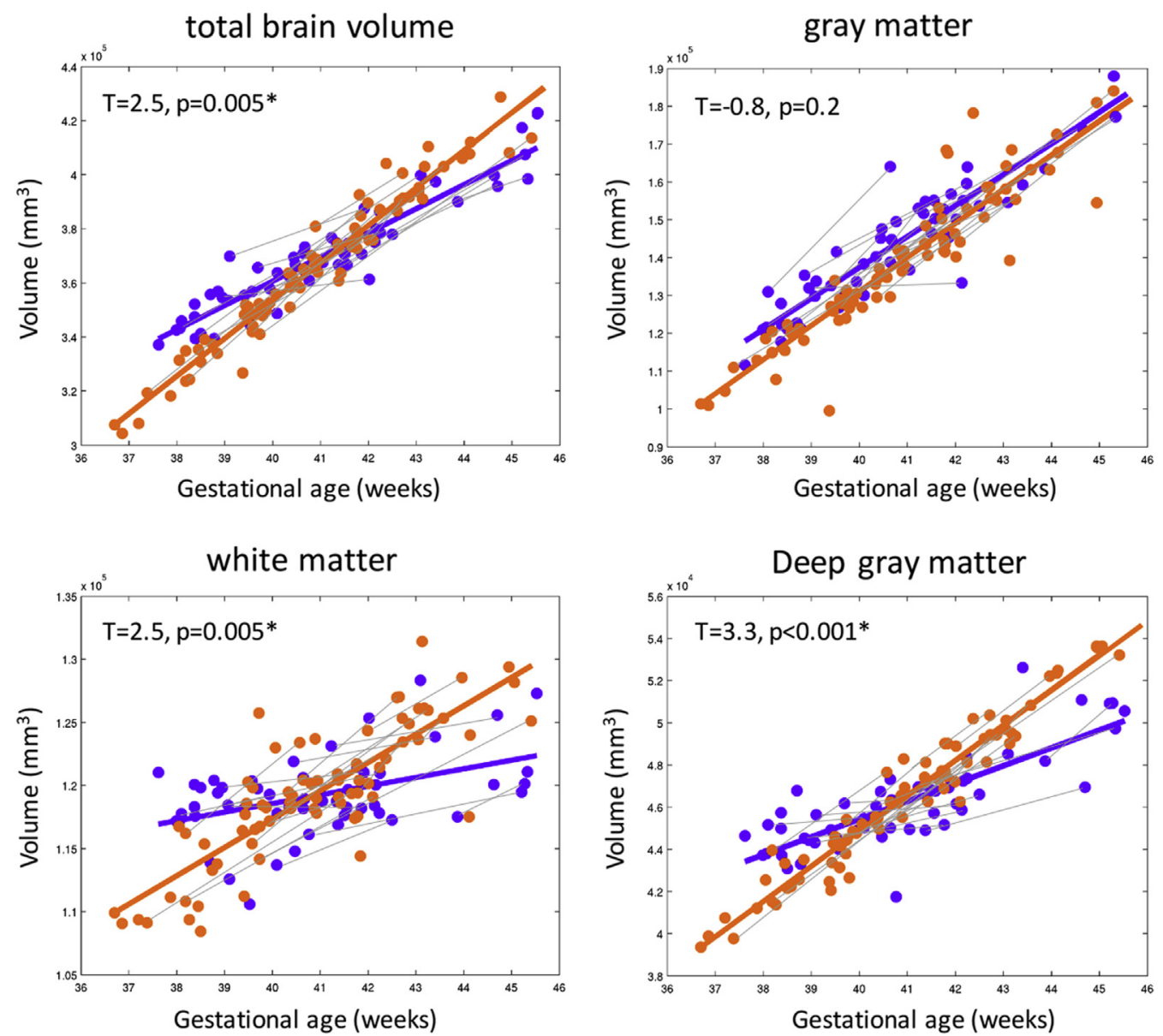

FIGURE E2. The rate of change in global and regional brain volumes by cardiac lesion after adjusting for the presence of brain injury. The x-axis represents the gestational age at the time of MRI, and the $y$-axis represents the volume in $\mathrm{mm}^{3}$. The plots include pre- and postoperative brain MRI measures with paired observations for each subject (gray line) and a best-fit line. Subjects with HLHS (purple line) have a slower rate of growth in total brain volume, white matter, and deep gray matter compared to those with d-TGA (orange line). *Significant after Bonferroni correction, $P<.006$. 